\title{
Wheelmap: the wheelchair accessibility crowdsourcing platform
}

\author{
Amin Mobasheri ${ }^{1 *}$ (D) Jonas Deister ${ }^{2}$ and Holger Dieterich ${ }^{2}$
}

\begin{abstract}
Crowdsourcing (geo-) information and participatory GIS are among the current hot topics in research and industry. Various projects are implementing participatory sensing concepts within their workflow in order to benefit from the power of volunteers, and improve their product quality and efficiency. Wheelmap is a crowdsourcing platform where volunteers contribute information about wheelchair-accessible places. This article presents information about the technical framework of Wheelmap, and information on how it could be used in projects dealing with accessibility and/or multimodal transportation.
\end{abstract}

Keywords: Wheelmap, OpenStreetMap, Open data, Crowdsourcing, VGl, Accessibility

\section{Introduction}

Wheelmap - a map for wheelchair-accessible places is an initiative of the Sozialhelden, a grassroots organisation from Berlin, Germany. On Wheelmap ${ }^{1}$ everyone from all over the world can find and add places and rate them by using a traffic light system. The map, which is available since 2010, shall help wheelchair users and people with mobility impairments to plan their day more effectively. Currently, more than 800,000 cafés, libraries, swimming pools, and many more public places have been captured. While the majority of the places which have been added so far are located in Germany, the mapping platform works globally, as it is based on OpenStreetMap (OSM). The Wheelmap interface is available in Arabic, Danish, German, Greek, English, Spanish, French, Icelandic, Italian, Japanese, Swedish, Turkish, Korean, and Polish.

Wheelchairs or purpose-built cars on the one hand, elevators and ramps on the other allow people with mobility impairments to plan their day independently to a great extent. But frequently, the last meters decide whether the trip to the cinema, beer garden or supermarket was worth the effort. Just one single step at the entrance can be an insurmountable obstacle, and this is where Wheelmap comes into play. Users

\footnotetext{
* Correspondence: a.mobasheri@uni-heidelberg.de

${ }^{1}$ GIScience research group, Institute of Geography, Heidelberg University, Heidelberg, Germany

Full list of author information is available at the end of the article
}

provide information for other users on how accessible a destination is. Thereby, the map contributes to an active and diversified lifestyle for wheelchair users. People with rollators or buggies benefit from this tool as well. Furthermore, the aim of Wheelmap is to make owners of wheelchair-inaccessible public places aware of the problem. They should be encouraged to reflect on and improve the accessibility of their premises.

As mentioned earlier, Wheelmap is based on OpenStreetMap, an open, editable map of the digital open source map of the world. Everyone can search for places and - provided they have been tagged - get information about how easily accessible the places are. Those who sign up as a user are able to add and rate new places. An easy traffic light system marks the wheelchair accessibility of a place: Green signifies an unrestricted access - e.g. because there are no steps or there is a permanent ramp, an elevator or other tools which allow the entrance. Places which are orangecolored have no toilets but might have a foldable ramp for example. Places which are red-colored are not accessible for wheelchair users. In general, the more people join Wheelmap and add places the more precise and informative the map gets.

Sozialhelden is an incorporated, not for profit society, located in Berlin, Germany. In addition to a small paid staff, it comprises a network of volunteers engaging in various activities regarding social justice. It 
is financed by taking part in contests, by receiving donations from public and private sponsors, and by supporting Wheelmap-related activities of social engagement.

The main objective is to gather information about the accessibility of public places (points of interest, POI). Everyone is invited to participate and provide their own tagging of places on an OSM-based map at the Wheelmap website.

\section{Background}

In the past decade, OpenStreetMap in particular, and Volunteered Geographic Information (VGI) in general, have gained special attention in various research projects. Such free and open datasets have been used in several application domains including routing and navigation [1-4], transportation studies [5, 6], urban and environmental challenges $[7,8]$, as well as disaster management [9].

So far, several efforts have been done regarding research studies specifically addressing accessibility issues using crowdsourced datasets. This is while there are still research gaps in developing an efficient framework for accessible transportation. For instance, in one of the early works, Prandi et al., [10] explored the potentials of the crowdsourcing communities in improving data access and services in the field of disable pedestrian mobility. Similar works have been followed by [11-19]. Among those, mPASS [19] presents a valuable mobile pervasive accessibility social sensing framework. mPASS collects data about urban and building accessibility to provide personalized paths. Their framework is similar to Wheelmap, besides the fact that Wheelmap only focuses on accessible points of interests (building, toilets, etc.), while mPASS also considers accessible routes. In another study, Salomoni et, al. [16] present the results of field trials with mobile applications that employing different gamification mechanisms. The authors conclude that some of the apps are able to drive users to provide more contributions. Further discussions on their study can be found at [16].

A main concern regarding using crowdsourced datasets such as OpenStreetMap is the level of quality they carry [20]. Several methods exists in assessing VGI data quality [21]. Among the studies carried out on using VGI data for urban accessibility, some of them evaluate, argue and enrich the fitness for purpose of this data source [22-24]. For instance, Mobasheri et, al. [23] evaluates the completeness of sidewalk information (as well as other relevant data for accessibility) in OpenStreetMap database and discusses the lack of data completeness with applying extrinsic and intrinsic data analysis. Wheelmap, among other possibilities, can be used by volunteers to enrich accessibility information in OpenStreetMap. Wheelmap has had a great influence in research projects. Several studies have used or cited Wheelmap as one of the main crowdsourcing platforms in the accessibility domain $[18,19,25-31]$. Hence, this study aims to introduce this platform for a better understanding and usage in future studies.

\section{Implementation}

\section{Wheelmap architecture}

Technically, Wheelmap currently comprises two major applications (Fig. 1). One is the publicly available platform for collaborative tagging, and the other is a platform for developers so that they can test new functionality and quality-check new data sources (e.g. sponsored data) before applying it to the live server.

Wheelmap provides a RESTful API to access and maintain the Wheelmap relevant data within OSM. The REST requests are to be authorized by an api_key, which can be obtained for a Wheelmap account. Such an account is based on a valid OSM account. Registration for Wheelmap is offered at Wheelmap's login website. ${ }^{2}$

\section{Resources at GitHub}

Public applications and components of Wheelmap are hosted as open source at GitHub.

Besides the standard web interface, the main applications for the mobile collaborative crowd-sourcing process are (Fig. 2):

- Wheelmap-android - An android app for Wheelmap, with full editing and search capabilities, direction pointers as well as a tablet version.

- Wheelmap-iPhone - An iOS version of Wheelmap

Recently added functionality includes helpful services such as:

- Users can add images to a place. Images can be added from either the camera or from the photo album.

- The "Get engaged" function shows unmarked places nearby.

- Users can share the link to any place on Wheelmap.org via Facebook, Twitter, email and SMS.

- Users can ask their network and friends via Facebook, Twitter and email if they know the wheelchair accessibility of an unmarked place and can tag it accordingly.

- The "Route" function shows users a route from their current position to a certain place on Wheelmap.org. In the list view, a user can see 


\section{Development}

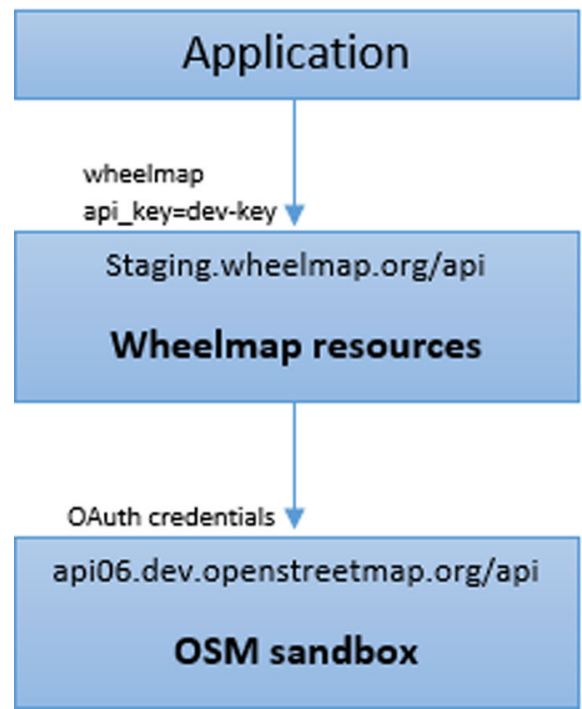

Live

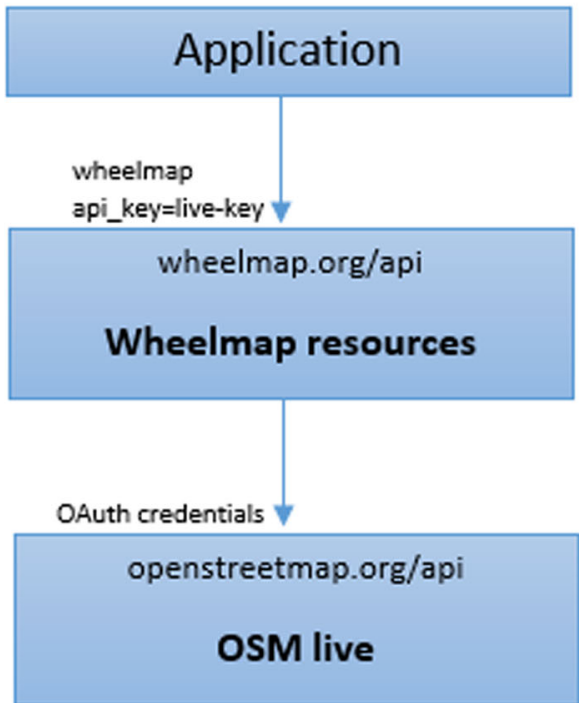

Fig. 1 The two Wheelmap applications with the Sandbox Developers' Playground (left), and the live operational platform (right)

how far away a place is from the current position of the user.

\section{Technologies}

Wheelmap, as well as OSM, is a Ruby on Rails ${ }^{3}$ application. Wheelmap therefore has created a ruby gem $^{4}$ client, named Rosemary, to easily access the current OpenStreetMap API. Furthermore, Maki ${ }^{5}$ is a point of interest icon set made especially for use with MapBox maps. Each icon is designed 3 times for 12, 18 , and 24 pixels wide/tall. Style files ${ }^{6}$ for ArcGIS $10.1+$ are available, including both Desktop and
Server versions, and standard and high-resolution versions of the PNG renders.

\section{The RESTful API}

The publicly documented RESTful API of Wheelmap is the most appropriate component to integrate Wheelmap data and facilities into new or foreign applications (Table 1).

Development and testing of applications is done against a sandbox branch of OSM, which is accessed by the RESTful Wheelmap API at http://staging.wheelmap.org/api/. Requests have to be authenticated and

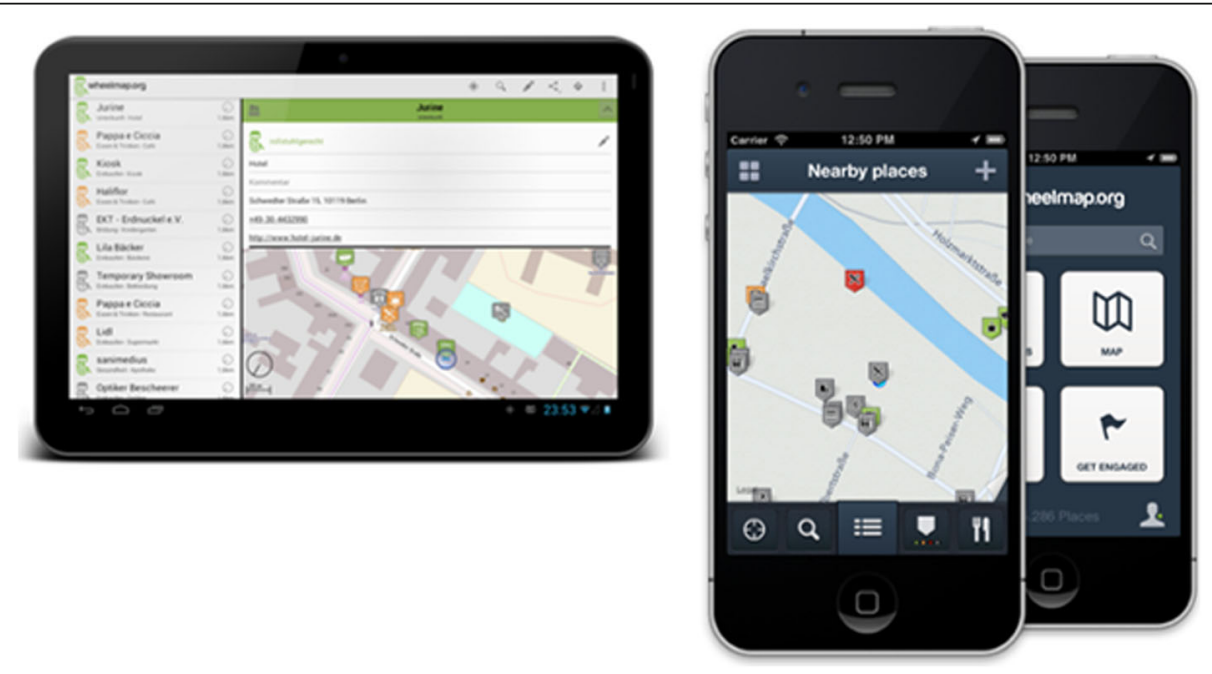

Fig. 2 Android app for tablet (left), iPhone app (right) 
Table 1 Overview of the resources of the RESTful API

\begin{tabular}{ll}
\hline API Docs & The documentation pages itself \\
\hline Resources & GET the base API URI - information about sub-resources. \\
Assets & GET the assets collection. \\
Categories & GET the categories collection. \\
Locales & GET a collection of all available locales. \\
Nodes & GET the nodes collection. \\
& - Filter nodes by a search term. \\
& - Filter nodes by a bounding box, \\
& - Filter nodes by a wheelchair status \\
& POST Create a new node. \\
& PUT Update an existing node. \\
& Update the wheelchair status of an existing node.
\end{tabular}

NodesTypes GET the node type collection.

This resource can also be nested within a category.

To just return the node types associated with the given category.

Users POST Authenticate a given user with email \& password and return the user's API key.

POST Declare that an authenticated user has wilfully accepted wheelmap.org's terms of usage and privacy policy.

Photos GET a collection of photos of a user or a node.

POST Upload a new Photo for the given node. thus authorized by a valid api_key. Changes made by the staging API at least appear in the recommended OSM sandbox for editing, which is accessible at http://api06.dev.openstreetmap.org/api. The effects of test runs appear at this site in the same way as they will appear in production at live OSM.

\section{Results and discussion}

\section{The tagging process}

Wheelmap's tagging process works similar to other crowdsourced tagging platforms [31-34]. When viewing a map, users will be shown all available POIs from a set of 12 categories (Fig. 3). Users may deselect those that they are not interested in and may contribute their own information by clicking on one of the icons. Clicking on a gray icon, i.e. one that has not yet received an accessibility value, results in a pop-up window where they can immediately choose one of the three presented options resulting in a subsequent green, orange or red colouring of the icon. Wheelmap users may only provide a new accessibility tag (colouring) without any constraints (e.g. no registration required) only when providing further information (via the "Details" button) Fig. 4a and c. This free process of tagging and re-tagging is proof of the high trust of the providers in the responsibility of the Wheelmap user community and would ease the process of data entry.

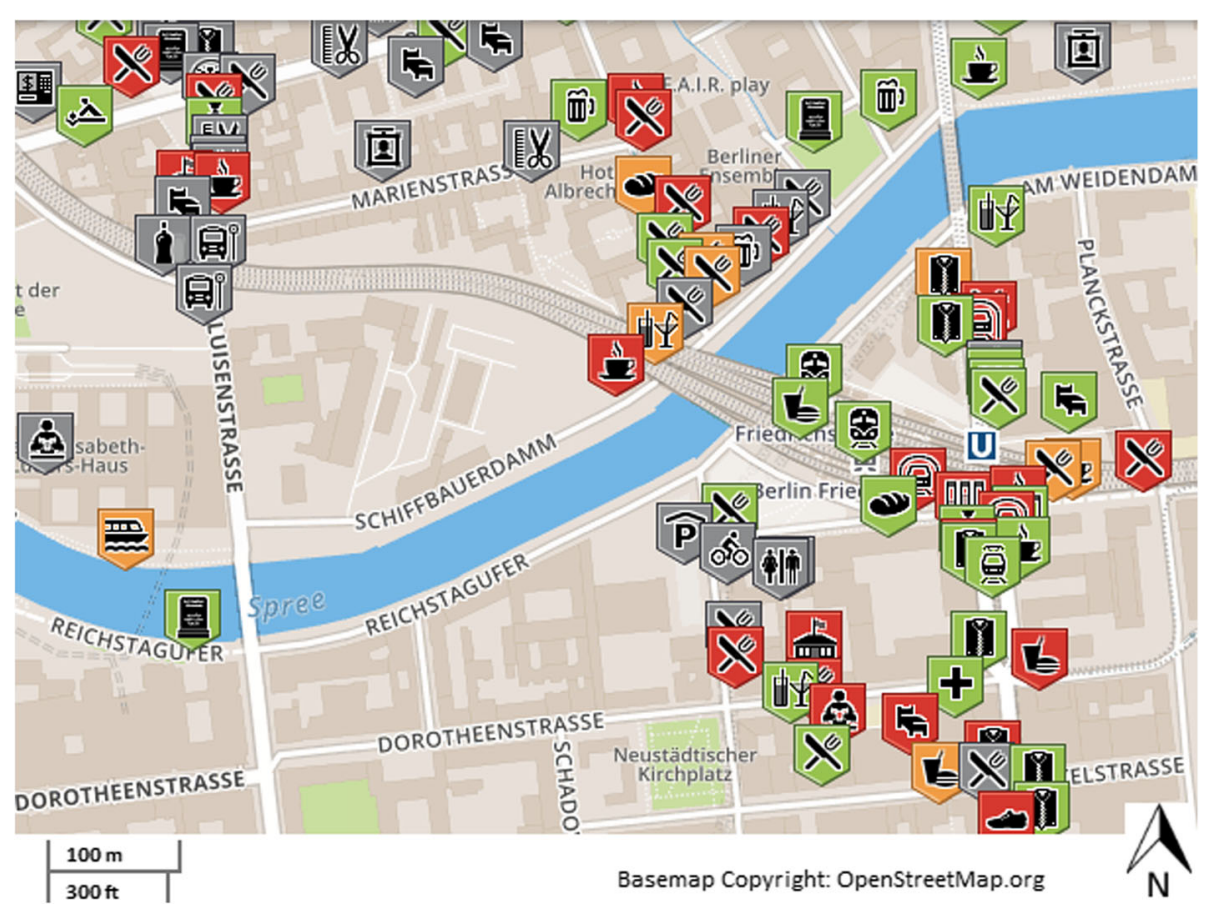

Fig. 3 A map showing POls in four different colours 


\section{a}

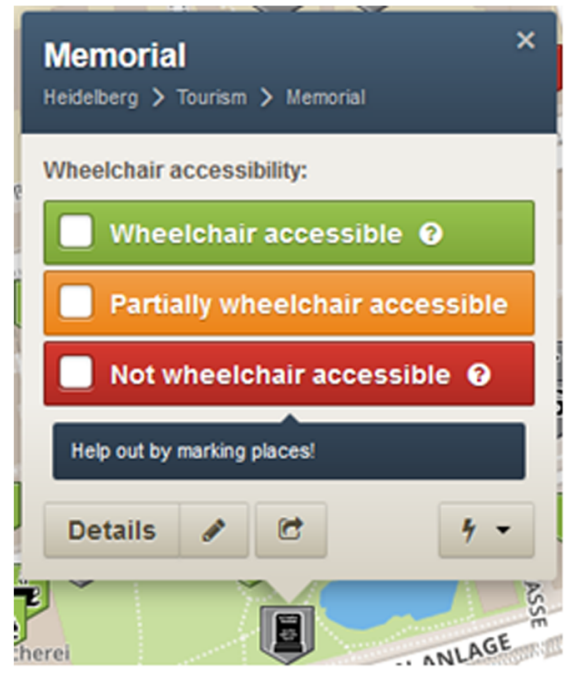

b

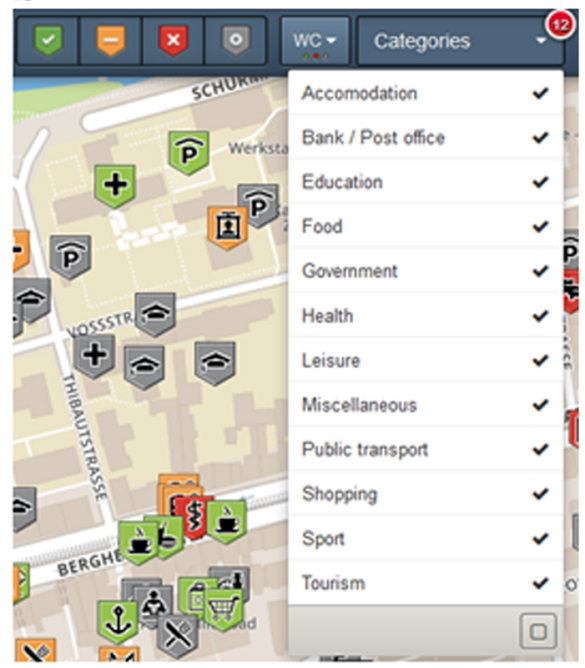

C

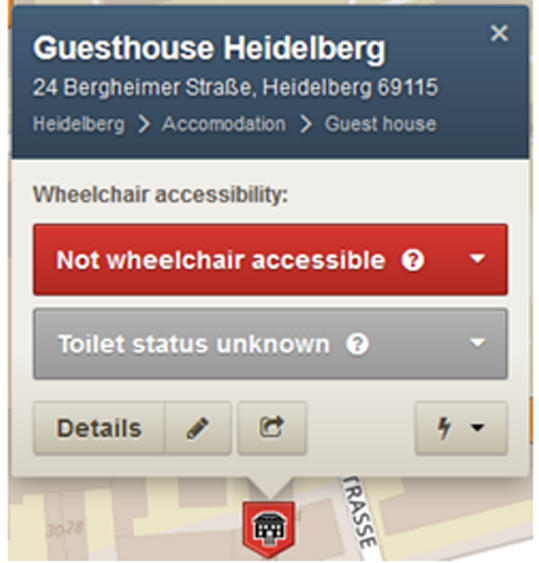

Fig. 4 a Tagging a new point (POI). b Selectable POI categories. c Further describe an already tagged point

Clicking on an already tagged non-gray icon returns a pop-up window as shown in Fig. 4 (c). This window shows the existing value for the tag, but allows the user to change it. This ease of changing tags will possibly have repercussions in later debates in the project about possible means of securing data quality and integrity. Interesting in this respect is also the button in the lower right corner of pictures (a) and (c) in Fig. 4. This is used in order to explicitly object to the existent assessment of a place, and to suggest another one. Geographical areas or particular users with exceptionally high rates of changes or change requests should give rise to major concerns and possibly actions to be taken from the provider's side.

Clicking on the "Details" button in the windows (a) and (c) in Figure triggers a window as depicted in Fig. 5. The photos of the tagged barbershop demonstrates why the shop is only "partially" accessible, e.g. because one has to take a small step in the entry passage ("there is a comment in red font by another user that the place is "not* accessible"). The right column of this window shows the location of the place on a map, its address, and a list of nearby alternative barbershops.

\section{Conclusion}

This article presents the framework and functionalities of Wheelmap - one of the promising existing platforms for the mobility impaired. Since its first release, Wheelmap has gained great interest and attention by the accessibility communities. Nowadays, Wheelmap has become the world's most extensive database on wheelchair accessible places; a database with more than 800,000 points of interest which have been rated by an active community gathered around the project. The Wheelmap project is one of several 


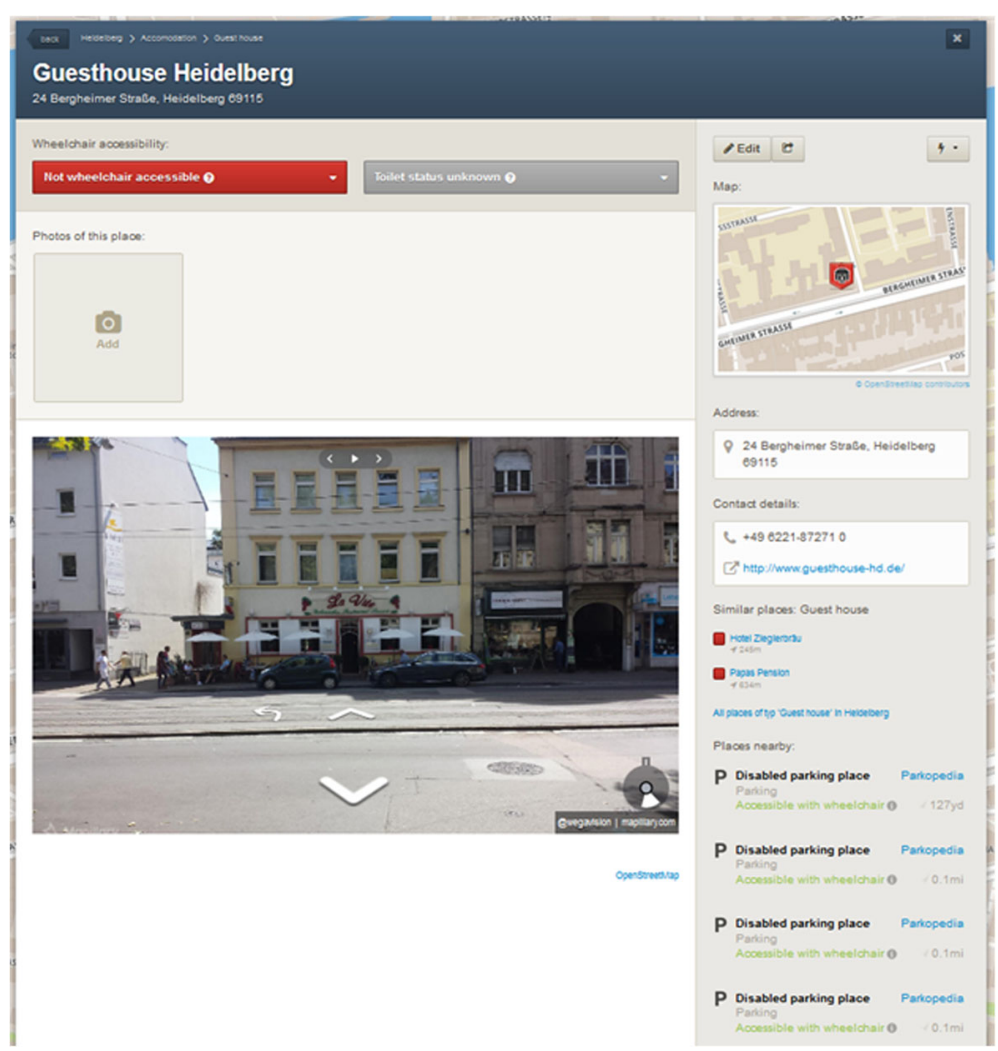

Fig. 5 Wheelmap tag info for a barbershop (category "partially accessible"), after selecting "Details" button in the menu of Fig. $4 a$

projects being run by a team at the non-profit organisation Sozialhelden. In order to ensure that this ambitious idea could grow into a successful project, Wheelmap's activity was designed for scalability from the very beginning. The Wheelmap team has won numerous national and international prizes. Among those are the Deutscher Bürgerpreis 2010 and World Summit Award Mobile 2012 by United Nations. It also received the Vodafone Accessibility Award 2011 from Neelie Kroes, formerly European Commissioner for Digital Agenda.

\section{Endnotes}

${ }^{1}$ http://www.wheelmap.org/

${ }^{2}$ http://wheelmap.org/users/sign_in.

${ }^{3}$ http://en.wikipedia.org/wiki/Ruby_on_Rails

${ }^{4}$ http://en.wikipedia.org/wiki/RubyGems

${ }^{5} \mathrm{http}: / /$ mapbox.com/tilemill/docs/guides/using-makiicons/

${ }^{6}$ https://GitHub.com/williamscraigm/makiArcGISStyle

${ }^{7}$ https://GitHub.com/sozialhelden/

${ }^{8}$ https://GitHub.com/sozialhelden/wheelmap-android

${ }^{9}$ https://GitHub.com/sozialhelden/wheelmap-iphone2

${ }^{10}$ https://GitHub.com/sozialhelden/rosemary

${ }^{11}$ http://wheelmap.org/de/api/docs

\section{Acknowledgements}

The authors would like to thank all the team members in Sozialhelden as well as project partners in European FP7 project CAP4Access, who have in any way supported and contributed to the Wheelmap project. We are also thankful to all the volunteers who use the service and provide accessibility information. We acknowledge OpenStreetMap project and its volunteers whom have provided the means and basic infrastructure for development of Wheelmap. We acknowledge the financial support of the Deutsche Forschungsgemeinschaft and Ruprecht-Karls-Universität Heidelberg within the funding programme Open Access Publishing.

\section{Funding}

Authors have received funding from the European Community's Seventh Framework Programme (FP7/2007-2013) under grant agreement No. 612096 (CAP4Access).

\section{Availability of data and materials}

All applications and components of Wheelmap are hosted as open source at GitHub. ${ }^{7}$ This includes Wheelmap-android, ${ }^{8}$ Wheelmap-iPhone, ${ }^{9}$ Rosemary, ${ }^{10}$ RESTful API of Wheelmap. ${ }^{11}$

\section{Authors' contributions}

AM has written the article. JD and HD have proofread and provided comments that improved the article. All authors read and approved the final manuscript.

Authors' information

Not applicable

Competing interests

The authors declare that they have no competing interests. 


\section{Publisher's Note}

Springer Nature remains neutral with regard to jurisdictional claims in published maps and institutional affiliations.

\section{Author details}

'GIScience research group, Institute of Geography, Heidelberg University, Heidelberg, Germany. ${ }^{2}$ Sozialhelden, Berlin, Germany.

Received: 18 October 2017 Accepted: 8 November 2017

Published online: 29 November 2017

\section{References}

1. Zhang X, Ai T. In advances in spatial data handling and analysis. In: How to model roads in OpenStreetMap? A method for evaluating the fitness-for-use of the network for navigation: Springer international publishing; 2015. p. $143-62$.

2. Bakillah M, Lauer J, Liang SH, Zipf A, Jokar Arsanjani J, Mobasheri A, Loos L. Exploiting big VGl to improve routing and navigation services. Big data techniques and technologies in geoinformatics. 2014:177-92.

3. Graser A, Straub M, Dragaschnig M. Is OSM good enough for vehicle routing? A study comparing street networks in Vienna. In: In progress in location-based services 2014: Springer international publishing; 2015. p. 3-17.

4. Bakillah, M., Mobasheri, A., Liang, S. H., \& Zipf, A.. Towards an efficient routing web processing service through capturing real-time road conditions from big data. In computer science and electronic engineering conference (CEEC), 2013, September 5th (pp. 152-155). IEEE.

5. Biljecki F, Ledoux H, Van Oosterom P. Transportation mode-based segmentation and classification of movement trajectories. Int J Geogr Inf Sci. 2013;27(2):385-407.

6. Sun Y, Fan H, Bakillah M, Zipf A. Road-based travel recommendation using geo-tagged images. Comput Environ Urban Syst. 2015;53:110-22.

7. Sun Y, Mobasheri A. Utilizing crowdsourced data for studies of cycling and air pollution exposure: a case study using Strava data. Int J Environ Res Public Health. 2017;14(3):274

8. Sun Y, Mobasheri A, Hu X, Wang W. Investigating impacts of environmental factors on the cycling behavior of bicycle-sharing users. Sustainability. 2017;9(6):1060.

9. Haworth B, Bruce E. A review of volunteered geographic information for disaster management. Geography Compass. 2015:9(5):237-50.

10. Prandi F, Soave M, Devigili F, De Amicis R, Astyakopoulos A. In: Collaboratively collected geodata to support routing service for disabled people, editor. Proceedings of the 11th international Symposium on Location-Based Services; 2014.

11. Duvall JA, Pearlman JL, Karimi HA. Development of route accessibility index to support wayfinding for people with disabilities. In: In Smart City $360^{\circ}$ Springer international publishing; 2016, October. p. 104-12.

12. Prandi, C., Salomoni, P., Roccetti, M., Nisi, V., \& Nunes, N. J. Walking with geo-zombie: a pervasive game to engage people in urban crowdsourcing In computing, networking and communications (ICNC), 2016 international conference on 2016, February.(pp. 1-5). IEEE.

13. Benner JG. Diffusion of collaborative maps about accessibility. In: IConference 2016 proceedings; 2016.

14. Mostafavi, M. A. MobilisIG, Development of a Geospatial assistive technology for navigation of people with motor disabilities. (2012).

15. Mirri, S., Prandi, C. \& Salomoni, P. Personalizing pedestrian accessible wayfinding with mPASS. In Consumer Communications \& Networking Conference (CCNC), 2016 13th IEEE annual 2016, January (pp. 1119-1124). IEEE.

16. Salomoni, P., Prandi, C., Roccetti, M., Nisi, V., \& Nunes, N. J.. Crowdsourcing urban accessibility:: Some preliminary experiences with results. In Proceedings of the 11th Biannual Conference on Italian SIGCHI Chapter 2015, September (pp. 130-133). ACM.

17. Karimi HA, Zhang L, Benner JG. Personalized accessibility map (PAM): a novel assisted wayfinding approach for people with disabilities. Ann GIS. 2014:20(2):99-108

18. Zipf A, Mobasheri A, Rousell A, Hahmann S. Crowdsourcing for individual needs-the case of routing and navigation for mobility-impaired persons European Handbook of Crowdsourced Geographic Information. 2016:325.

19. Prandi, C., Salomoni, P., \& Mirri, S.. mPASS: integrating people sensing and crowdsourcing to map urban accessibility. In consumer communications and networking conference (CCNC), 2014 IEEE 11th (2014, January) (pp. 591-595). IEEE.
20. Goodchild MF, Li L. Assuring the quality of volunteered geographic information. Spatial statistics. 2012;1:110-20.

21. Senaratne H, Mobasheri A, Ali AL, Capineri C, Haklay M. A review of volunteered geographic information quality assessment methods. Int J Geogr Inf Sci. 2017;31(1):139-67.

22. Rice MT, Paez Fl, Rice RM, Ong EW, Qin $\mathrm{H}$, Seitz CR, et al. Quality assessment and accessibility applications of crowdsourced geospatial data: a report on the development and extension of the George Mason University Geocrowdsourcing testbed: GEORGE MASON UNIV FAIRFAX VA; 2014

23. Mobasheri A, Sun Y, Loos L, Ali AL. Are crowdsourced datasets suitable for specialized routing services? Case study of OpenStreetMap for routing of people with limited mobility. Sustainability. 2017;9(6):997.

24. Mobasheri A. A rule-based spatial reasoning approach for OpenStreetMap data quality enrichment; case study of routing and navigation. Sensors. 2017;17(11):2498.

25. Ding, C., Wald, M., \& Wills, G.. A survey of open accessibility data. In proceedings of the 11th web for all conference 2014, April (p. 37). ACM.

26. Kozievitch, N. P., Almeida, L. D., da Silva, R. D., \& Minetto, R.. An alternative and smarter route planner for wheelchair users: exploring open data. In smart cities and green ICT systems (SMARTGREENS), 2016 5th international conference on. 2016, April (pp. 1-6). IEEE.

27. Benner JG, Oh JS. Accessibility cyberscapes and the digital divide. Proceedings of the Association for Information Science and Technology. 2014:51(1):1-4.

28. Voigt C, Dobner S, Ferri M, Hahmann S, Gareis K. In international conference on computers helping people with special needs. In: Community engagement strategies for crowdsourcing accessibility information: Springer international publishing; 2016, July. p. 257-64

29. von Lucke J, Große K, Harnessing the potential of IT-enabled collaboration-a classification for open government collaboration. conference for $\mathrm{E}$ democracy and open Governement; 2014, May. p. 219.

30. Zeng L, Kühn R, Weber G. Improvement in environmental accessibility via volunteered geographic information: a case study. In: Universal Access in the Information Society; 2016. p. 1-11.

31. Stein K, Schlieder C, der Weberei A. A geowiki for participatory mobility. In: Proc. 16th AGILE int. conf. on geographic information science. Digital proceedings; 2013. http://agile.gis.geo.tudresden.de/web/index.php/ conference/conference-2013.

32. Mooney P, Corcoran P. The annotation process in OpenStreetMap. Trans GIS. 2012;16(4):561-79.

33. Bakillah M, Mobasheri A, Rousell A, Hahmann S, Jokar J, Liang SH. Toward a collective tagging android application for gathering accessibility-related geospatial data in European cities. Parameters. 2014;10:21.

34. Rousell A, Hahmann S, Mobasheri A. A two-tiered approach to OSM data collection for novice users. In: The 19th AGILE International Conference on Geographic Information Science; 2016.

\section{Submit your manuscript to a SpringerOpen ${ }^{\circ}$ journal and benefit from:}

- Convenient online submission

- Rigorous peer review

- Open access: articles freely available online

- High visibility within the field

Retaining the copyright to your article

Submit your next manuscript at $>$ springeropen.com 